\title{
Research Article \\ Relativistic Short Range Phenomena and Space-Time Aspects of Pulse Measurements
}

\author{
Ezzat G. Bakhoum ${ }^{1}$ and Cristian Toma ${ }^{2}$ \\ ${ }^{1}$ Department of Electrical and Computer Engineering, University of West Florida, \\ 11000 University Parkway, Pensacola, FL 32514, USA \\ ${ }^{2}$ Faculty of Applied Sciences, Politechnica University, Hagi-Ghita 81, 060032 Bucharest, Romania \\ Correspondence should be addressed to Ezzat G. Bakhoum, ebakhoum@uwf.edu
}

Received 22 April 2008; Accepted 5 May 2008

Recommended by Carlo Cattani

Particle physics is increasingly being linked to engineering applications via electron microscopy, nuclear instrumentation, and numerous other applications. It is well known that relativistic particle equations notoriously fail over very short space-time intervals. This paper introduces new versions of Dirac's equation and of the Klein-Gordon equation that are suitable for short-range phenomena. Another objective of the paper is to demonstrate that pulse measurement methods that are based on the wave nature of matter do not necessarily correlate with physical definitions that are based on the corpuscular nature of particles.

Copyright (C) 2008 E. G. Bakhoum and C. Toma. This is an open access article distributed under the Creative Commons Attribution License, which permits unrestricted use, distribution, and reproduction in any medium, provided the original work is properly cited.

\section{Introduction}

The theory of special relativity plays a great role in particle physics. Now, particle physics is increasingly being linked to engineering applications, via electron microscopy, superconductivity, nuclear instrumentation, to name a few applications. Since relativistic formulae are at the heart of all such applications, then it becomes important to find ways to perform numerical computations related to localized (short-range) relativistic phenomena. For instance, it is well known that the relativistic version of Schrödinger's equation, namely, the Dirac equation, cannot normally be solved over a short interval because it always predicts that the velocity of the electron is equal to $c$, or the speed of light. In applications such as electron microscopy, it becomes therefore usually necessary to abandon the relativistic formulae and rely solely on the classical theory of electromagnetism. It is therefore clear that there is a need at the present time to formulate the Dirac and other relativistic equations in a manner that allows the computation of short-range phenomena. This is the first objective of this paper.

The second objective of this paper is to show that space-time measurements on closed-loop trajectories in special relativity and noncommutative properties of operators 
in quantum physics require a more rigorous definition of the method of measurement of interaction phenomena. The use of the least action principle, for instance, implies some logic definitions for measuring methods that are based on waves and for measuring methods that are based on the corpuscular aspects of matter. When measurement is applied to pulses, those logic definitions include considerations about a possible memory of previous measurements (space-time operators). Accordingly, a distinction exists between the set of existing space-time intervals and the set of measured space-time intervals (established using wave measurement methods and defined within limited space-time intervals).

\section{Relativistic short-range electron equation}

In this section, we will develop a version of Dirac's equation that is suitable for pulsed, short-range electron beams. We will rely on the recently introduced mass-energy equivalence relation $H=m v^{2}$ [1] (where $H$ is the total energy of the electron and $v$ is its velocity), which has proved to be effective in explaining short-range phenomena. First, a new Hamiltonian will be obtained. It will be then verified that the new Hamiltonian directly leads to the result that the velocity of the electron must be equal to $\pm v$, which is of course a result that is in sharp contrast with Dirac's result and which does agree with experimental observation. We will also verify that the spin magnetic moment term obtained by Dirac remains unchanged in the present formulation.

\subsection{The wave equation}

We will begin by describing briefly Dirac's approach for obtaining the relativistic wave equation and then proceed to derive the modified equation and hence the modified Hamiltonian. Dirac considered the mass of the particle as represented by its relativistic expression $m=m_{0} / \sqrt{1-v^{2} / c^{2}}$. If we square that expression and rearrange the terms, we get

$$
m^{2} c^{2}=m^{2} v^{2}+m_{0}^{2} c^{2}
$$

Multiplying by $c^{2}$, we get

$$
m^{2} c^{4}=m^{2} v^{2} c^{2}+m_{0}^{2} c^{4}
$$

But since $m c^{2}$ is the total energy according to Einstein, then we have

$$
H^{2}=p^{2} c^{2}+m_{0}^{2} c^{4}
$$

Hence,

$$
H=c \sqrt{p^{2}+m_{0}^{2} c^{2}}
$$

Since the term $p^{2}$ can be written as $\sum_{r} p_{r}^{2}$, where $p_{r}$ is a one-dimensional momentum component and $r=1,2,3$, we finally have

$$
H=c \sqrt{\sum_{r} p_{r}^{2}+m_{0}^{2} c^{2}}
$$


This was Dirac's total energy equation and was subsequently used to obtain the relativistic wave equation. To obtain the modified wave equation, we now proceed to multiply (2.1) by $v^{2}$, getting

$$
m^{2} c^{2} v^{2}=m^{2} v^{4}+m_{0}^{2} c^{2} v^{2}
$$

Using $H=m v^{2}$ as the total energy of the particle, we have, from the above expression,

$$
H^{2}=p^{2} c^{2}-m_{0}^{2} c^{2} v^{2}
$$

Now, since $v^{2}=\sum_{r} v_{r}^{2}$, where $v_{r}$ is a one-dimensional velocity component, (2.7) can equivalently be written as

$$
H=c \sqrt{\sum_{r} p_{r}^{2}-m_{0}^{2} \sum_{r} v_{r}^{2}}
$$

Equation (2.8) can be further simplified by noting that $v_{r}=p_{r} / m$. We finally have

$$
H=c \sqrt{\left(1-\frac{m_{0}^{2}}{m^{2}}\right) \sum_{r} p_{r}^{2}}
$$

Following Dirac's approach, if we let $\vec{p}_{0}$ be a vector defined as $\vec{p}_{0}=\vec{H} / c$, where $\vec{H}$ may be Hamiltonian of the form $\vec{H}=(H, 0,0)$, we will seek a wave equation that is linear in $\vec{p}_{0}$. We will take an equation of the most simple, basic form

$$
\left(\vec{p}_{0}-\sum_{r} \vec{p}_{r}\left[\alpha_{r}\right]\right) \psi=0
$$

This form can be sufficient without any additional terms if we do not impose any restrictions on the matrices $\left[\alpha_{r}\right]$. Dirac found that such matrices must be noncommuting, but it is obvious here that such matrices must also contain mass terms. Multiplying (2.10) by the vector $\left(\vec{p}_{0}+\sum_{r} \vec{p}_{r}\left[\alpha_{r}\right]\right)$, we get

$$
p_{0}^{2}-\left(\sum_{r} \vec{p}_{r}\left[\alpha_{r}\right]\right)^{2}=0
$$

Comparing this last expression with (2.9), we conclude that

$$
\left(1-\frac{m_{0}^{2}}{m^{2}}\right) \sum_{r} p_{r}^{2}=\left(\sum_{r} \vec{p}_{r}\left[\alpha_{r}\right]\right)^{2}=\sum_{r} \vec{p}_{r}\left[\alpha_{r}\right]^{2} \vec{p}_{r}^{T}+\sum_{j} \sum_{k} \vec{p}_{j}\left[\alpha_{j}\right]\left[\alpha_{k}\right] \vec{p}_{k}^{T},
$$

where $j, k=1,2,3$, and $j \neq k$. Accordingly, the matrices $\left[\alpha_{r}\right]$ must satisfy

$$
\left[\alpha_{r}\right]= \pm \sqrt{1-\frac{m_{0}^{2}}{m^{2}}}\left[\beta_{r}\right]
$$


where $\left[\beta_{r}\right]$ are matrices that must satisfy the following two conditions:

$$
\left[\beta_{r}\right]^{2}=I, \quad\left[\beta_{j}\right]\left[\beta_{k}\right]+\left[\beta_{k}\right]\left[\beta_{j}\right]=0
$$

Examples of such matrices were suggested by Dirac [2]. They might take the following forms among others:

$$
\left(\begin{array}{llll}
0 & 0 & 0 & 1 \\
0 & 0 & 1 & 0 \\
0 & 1 & 0 & 0 \\
1 & 0 & 0 & 0
\end{array}\right), \quad\left(\begin{array}{cccc}
0 & 0 & 0 & -i \\
0 & 0 & i & 0 \\
0 & -i & 0 & 0 \\
i & 0 & 0 & 0
\end{array}\right) .
$$

(Note that Dirac used $4 \times 4$ matrices to account for time as the fourth dimension. It was independently confirmed later that the minimum number of dimensions that will satisfy Dirac's theory is in fact four.)

Using the relativistic expression for $m$, the matrices $\left[\alpha_{r}\right]$ can now be written as

$$
\left[\alpha_{r}\right]= \pm \sqrt{1-\left(1-\frac{v^{2}}{c^{2}}\right)}\left[\beta_{r}\right]= \pm \frac{v}{c}\left[\beta_{r}\right] .
$$

Therefore, from (2.12) and (2.16), the vector Hamiltonian can be written as

$$
\vec{H}=c \vec{p}_{0}=c \sum_{r} \vec{p}_{r}\left[\alpha_{r}\right] \pm v \sum_{r} \vec{p}_{r}\left[\beta_{r}\right] .
$$

To check the modified theory, it can be now easily verified that the velocity component $\dot{x}_{1}$ will be given by

$$
\dot{x}_{1}=\left[x_{1}, \vec{H}\right]= \pm v .
$$

Unlike Dirac's result, this result is of course in agreement with experimental observation. It is important to note here that, mathematically, $\dot{x}_{1}$ is the "expected" value of the velocity. From (2.17), we can also see that the negative energy states are still preserved here.

\subsection{Motion of a charged particle in a magnetic field}

We now consider the motion of a charged particle in a magnetic field to obtain a formulation for the spin magnetic moment term that must appear in the final Hamiltonian (we assume the absence of an electrostatic field here). In the presence of a magnetic field, the change in the particle momentum $\Delta p$ that occurs as a result of the interaction with the field is given by [3]

$$
\Delta p=\frac{e}{c} A,
$$

where $e$ is the particle charge and $A$ is the magnitude of the vector magnetic potential. Adding that term to the momentum in (2.17) gives the Hamiltonian

$$
\vec{H}= \pm v \sum_{r}\left(\vec{p}_{r}+\frac{e}{c} \vec{A}_{r}\right)\left[\beta_{r}\right]
$$


By squaring (2.20), we get

$$
\frac{H^{2}}{v^{2}}=\sum_{r}\left[\left(\vec{p}_{r}+\frac{e}{c} \vec{A}_{r}\right)\left[\beta_{r}\right]\right]^{2}+\sum_{j} \sum_{k}\left(\vec{p}_{j}+\frac{e}{c} \vec{A}_{j}\right)\left[\beta_{j}\right]\left[\beta_{k}\right]\left(\vec{p}_{k}+\frac{e}{c} \vec{A}_{k}\right)^{T} \text {. }
$$

It is fairly easy to verify that the second term on the r.h.s. of the above expression must vanish since the $\vec{p}_{r}$ vectors commute and since the $\left[\beta_{r}\right]$ matrices satisfy condition (2.14). In Dirac's treatment of the subject, he was able to show that the following equation holds:

$$
\left[\left(\vec{p}_{r}+\frac{e}{c} \vec{A}_{r}\right)\left[\beta_{r}\right]\right]^{2}\left(\vec{p}_{r}+\frac{e}{c} \vec{A}_{r}\right)^{2}+\frac{\hbar e}{c}\left\|\vec{M}\left[\beta_{r}\right]\right\|
$$

where $\vec{M}=\operatorname{curl} \vec{A}$ is the magnetic field intensity vector. Equation (2.21) therefore becomes

$$
H^{2}=v^{2} \sum_{r}\left(\vec{p}_{r}+\frac{e}{c} \vec{A}_{r}\right)^{2}+v^{2} \frac{\hbar e}{c} \sum_{r}\left\|\vec{M}\left[\beta_{r}\right]\right\| .
$$

(Note that (2.23) is a scalar equation.) If we now let $H=m v^{2}$ and divide both sides of the equation by $2 m v^{2}$, we get

$$
\frac{1}{2} m v^{2}=\frac{1}{2 m} \sum_{r}\left(\vec{p}_{r}+\frac{e}{c} \vec{A}_{r}\right)^{2}+\frac{\hbar e}{2 m c} \sum_{r}\left\|\vec{M}\left[\beta_{r}\right]\right\| .
$$
becomes

If the particle is an electron, then $e$ is a negative quantity and the above equation

$$
\frac{1}{2 m} \sum_{r}\left(\vec{p}_{r}+\frac{e}{c} \vec{A}_{r}\right)^{2}=\frac{1}{2} m v^{2}+\frac{\hbar|e|}{2 m c} \sum_{r}\left\|\vec{M}\left[\beta_{r}\right]\right\| .
$$

Without the presence of the magnetic field, the 1.h.s. of (2.25) is reduced to

$$
\frac{1}{2 m} \sum_{r} p_{r}^{2}=\frac{p^{2}}{2 m}
$$

This is the same as $1 / 2 m v^{2}$. We can therefore conclude that the second term on the r.h.s. of (2.25) is the term that represents the interaction of the field with the electron magnetic moment. Hence the quantity $\hbar|e| / 2 m c$ is the spin magnetic moment coefficient. In general, we can withdraw here the following two important conclusions: (1) the modified theory fully yielded the classical expression of kinetic energy with the addition of the spin interaction term; and (2) the spin interaction term obtained here is the same as the one obtained by Dirac [2] (which is one Bohr magneton). The second conclusion is a confirmation that this part of Dirac's theory was correct. The first conclusion, however, shows a fact that was not apparent from Dirac's theory. Specifically, when $\vec{M}=0$ (i.e., when the particle is away from the magnetic field lines), (2.25) becomes

$$
\frac{1}{2 m} \sum_{r}\left(\vec{p}_{r}+\frac{e}{c} \vec{A}_{r}\right)^{2}=\frac{1}{2} m v^{2}
$$

This is a direct confirmation of the Aharonov-Bohm effect [4, 5]. Clearly, (2.27) shows that the components $p_{r}$ of the momentum will be altered while the kinetic energy remains constant. 


\section{Phase and group velocities of short-range electrons}

The concepts of the phase velocity and the group velocity are very important concepts that come into play when short-range phenomena are considered. For instance, de Broglie's work predicts that the phase velocity of a matter wave is given by the expression $c^{2} / v$, which is a very unrealistic assumption for short-range, slow electrons. We will attempt in this section to give a better explanation for that problem.

First of all, we must realize that there exists a number of phase velocities, not a single phase velocity. Now, it is well known mathematically that each phase velocity $v_{p}=\omega_{i} / k_{i}$ and that the group velocity $v_{g}=d \omega / d k$ (where $\omega$ is the angular frequency and $k=2 \pi / \lambda$ is the propagation constant). As was pointed out in [1], the two fundamental relationships of wave mechanics, $\lambda=h / p$ and $H=h v$, together make a statement about the total energy of a particle; that is, $H=(p \lambda) v=p u$, where $u$ is some velocity. The question here is what is $u$ exactly? Is it a phase velocity or a group velocity? Apart from the fact that $H=p u$ is a total energy equation, we must also note, since $H=\hbar \omega$ and $p=\hbar k$, that the equation leads to the relationship $\omega=k u$. Hence we must conclude that

$$
\frac{d \omega}{d k}=\frac{\omega}{k}=u
$$

This means that the group and the phase velocities are the same. This is the conclusion that we must hold as true for short-range phenomena. Let us now attempt to understand the origin of the problem. De Broglie's original derivation of the important relationship $\lambda=$ $h / p$ can be found in a number of standard references (see, e.g., [6]). Amazingly, as we will conclude, while the formula was correct, the approach that was used to derive it was not.

De Broglie started by assuming a wave function that describes a stationary particle of the form $\psi^{\prime}=\exp \left(i \omega^{\prime} t^{\prime}\right)$. By using the Lorentz transformation of time $t^{\prime}=\gamma\left(t-v x / c^{2}\right)$, then $\psi^{\prime}=\exp \left(i \gamma \omega^{\prime}\left[t-v x / c^{2}\right]\right)$. Since this equation (in principle) is a traveling wave equation, de Broglie then concluded that the quantity $c^{2} / v$ must represent the velocity of the wave in the observer frame. The rest of the derivation that leads to the formula $\lambda=h / p$ is then straightforward and consists of letting $H=h v=m c^{2}$ and substituting the product $\lambda v$ for the quantity $c^{2} / v$. As it is well known historically $[7,8]$, de Broglie later offered the hypothesis that $c^{2} / v$ is only a "phase" velocity and that the real, or "group" velocity is actually $v$ so that the particle and its associated wave would not part company. However, as we indicated, the problem with such a hypothesis is that it directly contradicts the simple conclusion in (3.1).

Let us try to understand the problem with the above approach that led to the indicated contradictions. The Lorentz transformation of time $t^{\prime}=\gamma\left(t-v x / c^{2}\right)$, which includes the coordinate $x$, strictly assumes that " $x$ " is only one geometrical point. From the viewpoint of a stationary observer, a traveling wave, in the observer's frame, cannot be described by one " $x$ " coordinate. The correct approach for including a traveling wave within the relativistic transformations is to assume first that the " $x$ " coordinate is equal to zero (and hence the time transformation will be $t^{\prime}=\gamma t$ ) and then write a true traveling wave equation in the observer frame, that is,

$$
\psi=\exp i(k x-\omega t)
$$

This was indeed the approach that was taken by Shrödinger and certainly this explains why Shrödinger's equation has been unquestionably successful. Now, by noting 
that $k=2 \pi / \lambda$ and $\omega=2 \pi v, \psi$ can be written as

$$
\psi=\exp i\left(\frac{2 \pi}{\lambda} x-\omega t\right)=\exp i \omega\left(\frac{2 \pi}{\lambda} \frac{x}{2 \pi \nu}-t\right)=\exp i \omega\left(\frac{x}{\lambda v}-t\right) .
$$

Assume first that the particle is moving with a velocity $v \ll c$ so that the relativistic effects can be ignored. In this case, ordinary (nonrelativistic) wave mechanics state that $\lambda v=$ $v$, or the wave velocity. Now, if the relativistic effect is to be included, then the wavelength $\lambda$ becomes $\lambda / \gamma$ (length contraction) and the frequency $v$ becomes $\gamma v$ (frequency shift). The result therefore is that $\lambda v$ is still equal to $v$. We can see, then, that the flaw in the original approach that led to the result $\lambda v=c^{2} / v$ was the incorrect use of the Lorentz transformation.

If we now follow the rest of de Broglie's derivation, but use $H=m v^{2}$ instead of $m c^{2}$, we have $H=m v^{2}=h v$, hence

$$
p=m v=\frac{h v}{v}=\frac{h v}{\lambda v}=\frac{h}{\lambda}
$$

which is of course de Broglie's well-known formula. De Broglie was aware that this relationship can be derived in a number of different ways, and for that reason he raised it to the level of a postulate. Concerning the approach that was used in deriving it, however, this is certainly one of the rare cases in science in which an incorrect derivation procedure still led to the correct result.

\section{A Klein-Gordon equation and a De Broglie dispersion relation for short-range electrons}

In this section, we present derivations for a modified Klein-Gordon equation and a modified de Broglie dispersion relation. The conclusions are: (1) in the case of a massless particle, the dispersion relation is the same as the original one; and (2) in the case of a massive particle, we still conclude that the phase and the group velocities are the same, that is, $v_{g}=v_{p}=v$.

\subsection{The Klein-Gordon equation}

The derivation of the Klein-Gordon equation starts with the usual relativistic expression (see [9])

$$
H^{2}=p^{2} c^{2}+m_{0}^{2} c^{4}
$$

If we now replace $H$ by $m c^{2}$ and $p$ by $m v$, we have

$$
m^{2} c^{4}=m^{2} v^{2} c^{2}+m_{0}^{2} c^{4}
$$

If we multiply this expression by $v^{2} / c^{2}$, we get

$$
m^{2} v^{2} c^{2}=m^{2} v^{4}+m_{0}^{2} v^{2} c^{2}
$$

If we now let $H=m v^{2}$, we finally have

$$
H^{2}=p^{2} c^{2}-m_{0}^{2} v^{2} c^{2} .
$$


This is a modified energy-momentum relationship and was in fact derived previously in [1]. Notice that the quantity $m_{0}^{2} v^{2}=p^{2}-H^{2} / c^{2}$. It is therefore a correct representation of the momentum vector $p^{\mu}$.

To obtain the modified Klein-Gordon equation, we start with the well-known relationship

$$
\nabla^{2} \psi=-\mathbf{k}^{2} \psi=-\frac{\mathbf{p}^{2}}{\hbar^{2}} \psi
$$

By substituting from (4.4) into (4.5) we have

$$
-\hbar^{2} \nabla^{2} \psi=\left(\frac{H^{2}}{c^{2}}+m_{0}^{2} v^{2}\right) \psi
$$

From Shrödinger's equation we have

$$
\frac{\partial^{2} \psi}{\partial t^{2}}=-\frac{H^{2}}{\hbar^{2}} \psi
$$

By substituting from (4.6) into (4.7) we finally get

$$
\frac{1}{c^{2}} \frac{\partial^{2} \psi}{\partial t^{2}}-\nabla^{2} \psi=\left(\frac{m_{0} v}{\hbar}\right)^{2} \psi
$$

This is the modified Klein-Gordon equation.

\subsection{De Broglie's dispersion relation}

In view of (4.7) and (4.5), the modified Klein-Gordon equation can be written as

$$
-\frac{1}{c^{2}}\left(\frac{\omega^{2} \hbar^{2}}{\hbar^{2}}\right) \psi=-\mathbf{k}^{2} \psi+\left(\frac{m_{0} v}{\hbar}\right)^{2} \psi, \quad \text { or }, \quad \omega^{2} \hbar^{2} \psi=c^{2} \hbar^{2} \mathbf{k}^{2} \psi-m_{0}^{2} c^{2} v^{2} \psi
$$

Hence, the modified de Broglie wave dispersion relation is

$$
\hbar^{2} \omega^{2}=c^{2} \hbar^{2} \mathbf{k}^{2}-m_{0}^{2} c^{2} v^{2}
$$

For $m_{0}=0$, we can see that the relation becomes $\hbar^{2} \omega^{2}=c^{2} \hbar^{2} \mathbf{k}^{2}$, which is of course the same as in the usual theory.

To obtain the group velocity, $\mathbf{v}_{g}=d \omega / d \mathbf{k}$, we differentiate the dispersion relation with respect to $\mathbf{k}$, getting (note that only the magnitudes of the vectors $\mathbf{p}$ and $\mathbf{k}$ will be represented)

$$
\hbar^{2} \omega \frac{d \omega}{d k}=c^{2} \hbar^{2} k-m_{0}^{2} c^{2} v \frac{d v}{d k}
$$

Since $p=m v=\hbar k$ and hence $m(d v / d k)=\hbar$, the above equation becomes

$$
\hbar^{2} \omega \frac{d \omega}{d k}=c^{2} \hbar^{2} k-m_{0}^{2} c^{2} \frac{\hbar^{2}}{m^{2}} k
$$

or

$$
\omega \frac{d \omega}{d k}=c^{2} k-\frac{m_{0}^{2}}{m^{2}} c^{2} k=c^{2} k\left(1-\left(1-\frac{v^{2}}{c^{2}}\right)\right)=k v^{2} .
$$


Hence,

$$
\frac{d \omega}{d k}=\left(\frac{k}{\omega}\right) v^{2}
$$

But since $d \omega / d k=v_{g}=v$, we then conclude that $\omega / k=v_{p}=v$. The group and the phase velocities are therefore the same.

\section{Logical aspects connected with space-time measurements}

After presenting basic aspects in physics from the relativistic point of view, we will present some logical aspects for basic principles in physics (the principle of constant light in vacuum in any reference system and the uncertainty principle in quantum theory). We will show that these principles make use in an implicit manner of terms which are defining also the conclusion. For example, the idea of constant light speed implies the use of a measuring method based on a clocks' synchronization performed using a supposed antecedent light signal transmitted and reflected toward the observer. In a similar manner, the uncertainty principle implies the existence of a measuring method for position or time correlated with a subsequent measurement for momentum or energy (measurements which also make use of position and time). Yet a logic definition of a physics principle cannot be based on the use of the same terms in both sides of it; like in the case of an algebraic calculus, the quantity to be determined must be finally placed in the opposite side of an equality, as related to the already known quantities joined in a mathematical operation. More precisely, we cannot define in a rigorous manner a certain term using the same term in the corresponding definition.

\subsection{Logical aspects of light speed constance principle}

The constant light speed principle (in vacuum) can be considered under the following form. It exists a quantity light speed in vacuum noted as c, which is constant for any observer inside an inertial reference system.

We can notice at first step that in an implicit manner the previous definition requires the existence of a measuring method for light speed in vacuum; any method for measuring a speed requires the use of time measurements (while $v=\Delta \mathbf{r} / \Delta t$ ). For our case (special relativity theory), the correspondence of time moments in different reference systems is based on a previous synchronization procedure implying an emission of light from an observer to another and a reflection of this light signal from the other observer to the first one. The reflection moment (considered as synchronization moment $\equiv$ zero moment) is considered by the first observer to take place at the middle of the time interval between the initial emission of light and the return of it. The whole chain implies that the use of a wave light LW appears in the definition of the light speed constance principle (in vacuum) under an explicit form (the notion of light speed), and it appears also under an implicit form (a previous synchronization based on light signals is required). From the formal logic point of view, this represents a contradiction [10]. A first attempt to solve it would be in taking into account the fact that the light speed measurement and the systems synchronization correspond to different time moments (the light wave considered for systems synchronization corresponds to the zero moment of time, while the light wave whose speed is considered in light speed constance principle corresponds to a subsequent moment of time). 
However, the use of such a set of different light waves (a light wave whose speed has to be measured and a previous pair of emitted-reflected light wave necessary for the synchronization procedure) implies the use of an extended time interval required by a light speed measurement as

$$
T_{m}=\left[t_{0}, t_{m}\right],
$$

where $T_{m}$ is the time interval required by a light speed measurement at $t_{m}$ time moment. But at next step we can notice that a speed corresponds to an almost instant moment of time, being defined as

$$
v=\lim _{t \rightarrow t_{m}} \frac{\Delta \mathbf{r}}{\Delta t}=\frac{d \mathbf{r}}{d t}
$$

This requires that the time interval required by a speed measurement must be infinitely small. Thus the time interval necessary for light speed measurement can be written as

$$
T_{m}=\left[t_{m}-\Delta t, t_{m}\right]
$$

which implies that the corresponding length interval $L_{T m}$ is infinitely small

$$
L_{T m} \longrightarrow 0
$$

But this is in contradiction with the previous consideration $T_{m}=\left[t_{0}, t_{m}\right]$. The corresponding timelength

$$
L_{T m}=t_{m}-t_{0} \gg 0
$$

can be much greater than zero. So the contradiction can be easily proved as

$$
L_{T M} \longrightarrow 0 \text { and in the same time } L_{T m} \gg 0 \text {. }
$$

From the intuitive point of view, this means that a light wave emitted in a certain reference system interacts in the most general case only on a limited time interval with another measuring reference system, the use of a previous procedure of emission-reflection for synchronization being impossible in practice. So the solution of such a contradiction (determined by implicit aspects of the terms used in definitions) must be found by taking into consideration other properties of physics entities involved in definition; see also [11].

\subsection{Logical aspects of uncertainty principle in quantum mechanics}

If we study the uncertainty principle in quantum mechanics, we can notice quite similar aspects. According to this principle, a measurement performed with a greater accuracy upon space or time coordinates for a quantum particle must generate a greater error upon a subsequent measurement for momentum or energy according to

$$
\Delta x \Delta P_{x} \geq \frac{h}{4 \pi}
$$


or

$$
\Delta t \Delta E \geq \frac{h}{4 \pi}
$$

But the existence of a measuring method for position or time is correlated with a subsequent measurement for momentum or energy (measurements which also make use of position and time).

It can be noticed that a term (a space-time measurements) is explained using (in an implicit manner) the same term at a subsequent moment of time. Without being a contradiction (like in the case of light speed constance principle), it still remains a recurrent definition. In the same manner presented for special relativity, we can take into consideration the different moments of time for space-time measurements. Yet the fact that (in an implicit manner) the principle requires the use of a measurement performed at a later time moment generates another logical problem. Can a space-time measurement performed at a certain moment of time be influenced by previous space-time measurements performed upon the same quantum particle? When a space-time measurement belongs to the class of spacetime coordinates measurement, and when it belongs to the class of momentum or energy measurements (performed in an indirect manner using also space-time measurements)? Under which circumstances a measurement can be considered as an initial action (in this case its accuracy can be greater) or as a subsequent action (its accuracy having to be less than a certain value, according to Heisenberg relation)?. The time always appears in quantum mechanics, while two physical quantities cannot be measured exactly at the same moment of time.

So a space or time measurement performed at a certain time moment belongs to the class of subsequent indirect methods for measuring momentum or energy (having as a consequence a limited accuracy), or to the class of direct methods for measuring space or time (having a possible greater accuracy). A rigorous classification according to certain patterns should be made; see also [12], taking into consideration similarities in fundamental physics laws [13].

\subsection{Different-scale system properties used for explaining logical aspects of pulse measurements}

This problem suggests also a possible solution: if we continue our analysis of terms involved in measuring procedures, we can notice that both basic principles (light speed constance principle and uncertainty principle) use the term of measuring method. In an implicit manner, the terms reference system (for special relativity theory) and measuring system (for quantum theory) appear. Yet a measuring system implies the fact that it is not affected by the measuring procedure (otherwise, the physical quantity having to be measured would possess different values, depending on this interaction). So a first conclusion appears: the measuring system must be defined at a much larger scale than the body or the wave which interacts with it. The different scale system properties must be taken into consideration from the very beginning so as to put them into correspondence with

(i) the class of reference systems, which are not affected by interaction (where wave trains similar to wavelets can appear [14]); 
(ii) the class of transient phenomena which undergo specific interactions (such transient phenomena can be represented as solitary waves, while estimations for the space coordinates for the source of the received wave-train based on space relations are not suitable for this purpose. As a further consequence, the constance light speed principle appears as a simple generation of another light wave when a received wave train arrives in the material medium of the observer reference system, and the uncertainty principle appears as a spread of a wave corresponding to a quantum particle by the measuring system, according to a kind of Fourier transformation performed on limited space and time intervals (the aperture and a certain working period). Thus logical aspects of the definitions of basic principles in physics (implying measurements of pulse parameters) can be explained in a rigorous manner.

\section{Aspects connected with measurements on a set of pulses}

\subsection{Measurements on a set of pulses received on adjoining space-time intervals. Synchronization aspects}

We will justify the previous considerations by presenting the case of measurements for sequence of pulses received on adjoining space-time intervals. As it is known, the special relativity theory considers that the Lorentz formulae describe the transformation of the space-time coordinates corresponding to an event when the inertial reference system is changed. These formulae are considered to be valid at any moment of time after a certain synchronization moment (the zero moment) irrespective to the measuring method used. However, there are some problems connected to the use of mechanical measurements on closed-loop trajectories. For example, let us consider that at the zero moment of time, in a medium with a gravitational field which can be neglected (the use of the Galileean form of the tensor $g_{i k}$ being allowed) two observers are beginning a movement from the same point of space, in opposite directions, on circular trajectories having a very great radius of curvature. After a certain time interval, the observers are meeting again in the same point of space. For very great radii of curvature, the movements on very small time intervals can be considered as approximative inertial (as in the case of the transverse Doppler effect, where the time dilation phenomenon was noticed in the earth reference system which is approximative inertial on small time intervals). The Lorentz formulae can be applied on a small time interval $\Delta t(1)$ measured by one of the observers inside his reference system, and it results (using the Lorentz formula for time) that this interval corresponds to a time interval

$$
\Delta t^{\prime}(1)=\frac{\Delta t(1)}{\sqrt{1-v(1)^{2} / c^{2}}}
$$

in the reference system $S_{2}$ of the other observer, which moves with speed $v(1)$ as related to the reference system $S_{1}$ on this time interval. So the time dilation phenomenon appears. If each observer considers the end of this time interval $\left(\Delta t(1)\right.$ or $\left.\Delta t^{\prime}(1)\right)$ as a new zero moment (using a resynchronization procedure), the end of the second time interval $\Delta t$ (2) (with the new zero moment considered as origin) will correspond to a time moment

$$
\Delta t^{\prime}(2)=\frac{\Delta t(1)}{\sqrt{1-v(2)^{2} / c^{2}}}
$$


measured in the other reference system $S_{2}$ which moves with speed $v(2)$ as related to system $S_{1}$ on the time interval $\Delta t^{\prime}(2)$ (with the new zero moment considered as origin). As related to the first zero moment (when the circular movement has started) the end of the second time interval appears at the time moment

$$
t_{2}=\Delta t(1)+\Delta t(2)
$$

For the observers situated in reference system $S_{1}$, and at the time moment

$$
t^{\prime}(2)=\Delta t^{\prime}(1)+\Delta t^{\prime}(2) \frac{\Delta t(1)}{\sqrt{1-v(1)^{2} / c^{2}}}+\frac{\Delta t(2)}{\sqrt{1-v(2)^{2} / c^{2}}}
$$

for the other observer.

Due to the fact that

$$
\begin{aligned}
& \Delta t^{\prime}(1)>\Delta t(1), \\
& \Delta t^{\prime}(2)>\Delta t(2),
\end{aligned}
$$

it results that

$$
t^{\prime}(2)=\Delta t^{\prime}(1)+\Delta t^{\prime}(2)>\Delta t(1)+\Delta t(2)=t(2)
$$

and thus a global time dilation for the time interval $\Delta t(1)+\Delta t(2)$ appears. The procedure can continue, by considering the end of each time interval

$$
\Delta t(1)+\Delta t(2)+\cdots+\Delta t(i)
$$

as a new zero moment, and so it results that on all the circular movement period, a time moment

$$
t(k)=\sum_{i=0}^{k} \Delta t(i)
$$

(measured by the observer in reference system $S_{1}$ ) corresponds to a time moment

$$
t^{\prime}(k)=\sum_{i=0}^{k} \Delta t^{\prime}(i)=\sum_{i=0}^{k} \frac{\Delta t(i)}{\sqrt{1-v_{i}^{2} / c^{2}}}
$$

(measured by the observer situated in reference system $S_{2}$ ) which implies

$$
t^{\prime}(k)>t(k)
$$

By joining together all these time intervals $\Delta t(i)$ we obtain the period of the whole circular movement $T$. While the end of this movement is represented by the end of the time interval $\Delta t(N)$ in the reference system $S_{1}$, it results that $T$ can be written under the form

$$
T=t(N)=\sum_{i=0}^{N} \Delta t(i)
$$


(considered in the reference system $S_{1}$ ) and it results also that this time moment (the end of the circular movement) corresponds to a time moment

$$
T^{\prime}=t^{\prime}(N)=\sum_{i=0}^{N} \Delta t^{\prime}(i)
$$

measured in the reference system $S_{@}$. While

$$
\Delta t^{\prime}(i)=\frac{\Delta t(i)}{\sqrt{1-v(i)^{2} / c^{2}}}>\Delta t(i),
$$

it results

$$
T^{\prime}>T \text {. }
$$

If the time is measured using the age of two twin children, it results that the twin in reference system $S_{2}$ is older than the other in reference system $S_{1}$, (having a less mechanical resistance of bones) and it can be destroyed by it after both observers stop their circular movements. However, the same analysis can be made starting from another set of small time intervals $\Delta_{n} t^{\prime}(i)$ considered in the reference system $S_{2}$ which corresponds to a new set of time intervals $\Delta_{n} t(i)$ considered in the reference system $S_{2}$ (established using the same Lorentz relation) and finally it would result that the period of the circular movement $T^{\prime}$ measured in system $S_{2}$ corresponds to a period $T$ greater than $T^{\prime}$ considered in reference system $S_{1}$. If the time is measured using the age of two twin children, it results that the twin in reference system $S_{1}$ is older than the other in reference system $S_{2}$, (having a less mechanical resistance of bones) and it can be destroyed by it after both observers stop their circular movements. But this result is in logic contradiction with the previous conclusion, because a man cannot destroy and in the same time be destroyed by another man [15].

As a first attempt of solving this contradiction, one can suppose that Lorentz formulae are valid only for electromagnetic phenomena (as in the case of the transversal Doppler effect) and not in case of mechanical phenomena. But such a classification is not a rigorous classification, being not suitable for formal logic. In next section, we will present a more rigorous classification of phenomena used in space-time measurements, which can be used for gedanken experiments using artificial intelligence based on formal logic.

\subsection{Classification of space-time measurement methods based on memory of previous measurements}

The logical contradiction presented in previous section appeared due to the fact that an element with internal memory has been used. The indication of this element has not been affected by the resynchronization procedure. In modern physics such an element with internal memory is connected with the corpuscular aspect of matter with a body. On the contrary, a measuring procedure based on an electromagnetic or optic wave train is a transient phenomenon. The synchronization of clocks is possible only after the wave-train arrives at the observer. Excepting a short time interval after the reception the received wave train does not exist inside the observer medium, so there is not any space area where a physical quantity which characterizes the wave to cumulate. That is the reason why a correct solution of the twins paradox must be based not on the association of electromagnetic (or optic) 
phenomena with the Lorentz formulae, but on the association of the Lorentz formulae with wave phenomena describing the propagation of a wave inside the observers reference systems. The wave class is more general than the class of electromagnetic and optic waves (we can mention the wave associated with particles in quantum mechanics). Besides, in the most general case, the interaction between two reference systems appears under the form of a field, not under the form of a material body. Moreover, this aspect implies an intuitive interpretation for the dependence of the mass of a body inside a reference system.

Using the formal logic, all we have shown can be presented in a rigorous manner.

(a) We define the notion of "propagation" phenomenon in two inertial reference systems (the system where the event takes place and the system where a signal generated by the event is noticed) as a phenomenon having a finite existence inside the reference system, the number of intervals being finite.

(b) We define the notion of corpuscle inside a certain reference system as a phenomenon which can possess an unlimited evolution in time and space inside the reference system; it can be also said that the phenomenon has its own existence, it exists by itself.

(c) We define the emission of a wave-train $U_{e}$ in a reference system and its transformation in another train when it interacts with the observers medium

Definition 6.1. There exist an area $S_{0 e}$ and a time interval $T_{0 e}$ in the reference system where the emission takes place so that

$$
F_{u e}\left(S_{0 e}, T_{0 e}\right) \neq 0, \quad F_{u e}\left(S_{0 e}, t\right)=0 \quad \text { for } t \notin T_{0 e} .
$$

There exist a space area $S_{0 r}$ and a time interval $T_{0 r}$ in the observer reference system, and a relation $\operatorname{Tr}$ so that

$$
\begin{gathered}
F_{u r}\left(S_{0 r}, T_{0 r}\right)=\operatorname{Tr}\left[F_{u e}\left(S_{0 e}, T_{0 e}\right)\right] \\
F_{u r}\left(S_{0 r}, T_{0 r}\right) \neq 0, \quad F_{u r}\left(S_{0 r}, t\right)=0 \quad \text { for } t \notin T_{0 r} .
\end{gathered}
$$

(d) We define the transformation of a sequence of received pulses $\Sigma_{k} U e_{k}$ in a sequence $\Sigma_{k} U r_{k}, k=1 \cdots n$ after interaction with the observers reference system, by considering that each pulse (wave-train) is transformed in an independent manner by the material medium of the observer reference system, according to its specific Lorentz transformation

Definition 6.2. Consider

$$
\begin{aligned}
& U r_{k}=L_{k}[U e]_{k \prime} \\
& \Sigma_{k} U e_{k}=\Sigma_{k} U r_{k},
\end{aligned}
$$

where $L_{k}$ represents the Lorentz transformation performed upon the $U e_{k}$ wave by the system with the interaction moment of this wave with the material medium of the observer considered as zero moment of time (synchronization moment) for the Lorentz transformation $L_{k}$.

(e) We define the interaction between a sequence of pulses and the material body of the observer reference system (a corpuscle) as an interaction function Int between the material medium and each transformed pulse $U r_{k}$ corresponding to a received pulse $U e_{k}$, the mass $m$ of the body measuring the influence of the received wave-train $U e_{k}$ upon the body. 
Definition 6.3. Consider

$$
\frac{1}{m}=\operatorname{Int}\left[U r_{k}\right]=\operatorname{Int}\left[L_{k}(U e)_{k}\right]
$$

When Lorentz transformation $L_{k}$ does not generate a pulse $U r_{k}$ (e.g., when the relative speed between the material body and the wave is equal to $c$, the speed of light in vacuum), the mass $m$ is equal to $\infty$, which means that no interaction due to the received pulse $U e_{k}$ exists (an idea which connects the notion on infinite mass with the absence of interaction). So $m=\infty$ for a body inside a reference system $S$ shows that we cannot act upon the material body using wave pulses emitted in system $S$; however, changes in the movement of the body (considered in system $S$ ) due to other external forces seem to be allowed.

By interaction with a certain material medium, each pulse is transformed according to Lorentz formulae, and the modified parameters of each pulse must replace the previous informations in the memory cells.

\section{Associating a certain wave train to Lorentz transformation}

\subsection{The necessity for associating a wave function to the Lorentz transformation}

The Lorentz transformation is usually represented as a matrix $L$ which acts upon a quadridimensional column vector $r$ having the components $r_{1}=x, r_{2}=y, r_{3}=z, r_{4}=$ ict, resulting in another quadridimensional vector $r^{*}$ having the components $r_{1}^{\prime}=x^{\prime}, r_{2}^{\prime}=y^{\prime}, r_{3}^{\prime}=$ $z^{\prime}, r_{4}^{\prime}=$ ict' $^{\prime}$, where $x, y, z, t$ are the space-time coordinates corresponding to a certain event in an inertial reference system $S$, and $x^{\prime}, y^{\prime}, z^{\prime}, t^{\prime}$ are the space-time coordinates corresponding to the same event measured in an inertial reference system $S^{\prime}$ which moves with velocity $v$ (a vector) against the system $\mathrm{S}$. This means

$$
r^{\prime}=L(v) r
$$

All time moments are considered after a synchronization moment (when the clock indications in the reference systems are set to zero). The velocity $v$ defines the matrix $L$, and the result is considered not to depend on the measuring method used. But let us consider that the velocity $v$ has two components $v_{x}$ and $v_{y}$ oriented along the $O x$ axis (for $v_{x}$ ) and along the $O y$ axis (for $v_{y}$ ) and let us consider also that the event taking place in the reference system $S$ is first observed in a reference system $S_{1}$ which moves with velocity $v_{x}$ as against the system $S$ :

a set of space-time coordinates $\left(x_{1}, y_{1}, z_{1}, t_{1}\right)$ will be established for the event. Then the event having the space-time coordinates $\left(x_{1}, y_{1}, z_{1}, t_{1}\right)$ in system $S_{1}$ is observed in the reference system $S^{\prime}$ which moves with velocity $v_{y}$ (the projection of $v$ along the $O y$ axis) against the reference system $S$ (this relative speed being measured in system $S$ ). That corresponds to a relative speed

$$
v_{y}(c)=\frac{v_{y}}{\sqrt{1-v_{x}^{2} / c^{2}}}
$$

between the systems $S$ and $S^{\prime}$ (due to the kinematics law of addition of speeds in special relativity theory). Thus will result in the cuadridimensional vector $r^{\prime}$ (having the components $x^{\prime}, y^{\prime}, z^{\prime}$, ict'), measured in system $S^{\prime}$, under the form

$$
r^{\prime}=L\left(v_{y}(c)\right) L\left(v_{x}\right) r .
$$


But we can also consider that the event having the space-time coordinates $x, y, z, t$ in system $S$ is first observed in a reference system $S_{2}$ which moves with velocity $v_{y}$ (the projection of velocity $v$ along the $O y$ axis) as against system $S$; a set of space-time coordinates will be established for the event. Then this event having the space-time coordinates $x_{2}, y_{2}, z_{2}, t_{2}$ in system $S_{2}$ is observed in the reference system $S^{\prime}$ which moves with velocity $v_{x}$ (the projection of velocity $v$ along the $O x$ axis) against the reference system $S_{2}$, the velocity $v x$ being measured in the reference system $S$. That corresponds to a relative speed

$$
v_{x}(c)=\frac{v_{x}}{\sqrt{1-v_{y}^{2} / c^{2}}}
$$

between the systems $S^{\prime}$ and $S_{2}$ (due to the same kinematics law of addition of speeds in special relativity). Thus will result in the space-time coordinates $x^{\prime}, y^{\prime}, z^{\prime}, t^{\prime}$ measured in system $S^{\prime}$ under the form

$$
r^{\prime}=L\left(v_{x}(c)\right) L\left(v_{y}\right) r
$$

Using the explicit form of Lorentz transformation for the case when the relative speed has the direction of one of the axes of coordinates, it can be easy shown that

$$
L\left(v_{y}(c)\right) L\left(v_{x}\right) r \neq L\left(v_{x}(c)\right) L\left(v_{y}\right) r .
$$

This shows that the coordinates measured for the event in $S^{\prime}$ reference system depends on the succession of transformations. This aspect is similar to the noncommutative properties of operators in quantum theory [16]. It implies that in the case of special relativity we must define a vector of state (a wave-function) upon which the Lorentz transformation acts. Thus the Lorentz transformation can be considered as a physical transformation which modifies a certain wave function inside a reference system. Taking into account the fact that usually we receive information under the form of electromagnetic (or light) wave trains (the emission of these wave trains corresponding to the event) and taking also into account the fact that the time-dilation phenomenon (a consequence of Lorentz transformation) was first time observed for light wave trains (the transverse Doppler effect) it results that in the most general case this wave function must be associated to the wave-function of the received light wave train. As a consequence of the previous statement, it results that a Lorentz transformation $L$ must be always put in correspondence with a pair $(S, \varphi), S$ representing a certain material reference system which acts upon a wave train having the state-vector $\varphi$. So the Lorentz transformation must be written under the form $L_{S}(\varphi)$; in the most general case $L$ is the Lorentz matrix and $\varphi$ is a vector or a higher-order tensor which describes the field. For an electromagnetic wave, the field can be described using the cuadridimensional vector $A$. The action of the matrix $L_{S}$ consists in a general transformation

$$
\varphi(x, y, z, t) \longrightarrow \varphi^{\prime}\left(x^{\prime}, y^{\prime}, z^{\prime}, t^{\prime}\right)=L_{S} \varphi(x, y, z, t)
$$

where the values of $\varphi$ are modified according to the transformation rules of vectors and tensors (e.g., $A^{\prime}=L A$ for an electromagnetic wave described by the cuadrivector $A$ ) and in the change of the space-time coordinates $(x, y, z, t)$ into $\left(x^{\prime}, y^{\prime}, z^{\prime}, t^{\prime}\right)$ according to the formula

$$
r^{\prime}=L_{S} r
$$


$r$ representing the cuadridimensional vector of coordinates. We have to point the fact that in all these formulae $\varphi(x, y, z, t)$ represents the value $\varphi$ would have possessed in the absence of the interaction with the observer material medium; the space-time origin must be considered in the point of space and at the moment of time where the wave first time interacts with the observer material medium (in a similar way with the aspects in quantum mechanics, where all transformations are acting after the interaction with the measuring system). This interpretation can solve the contradictions appearing in case of movements on closed-loop trajectories (the twins paradox) in a very simple manner. The Lorentz transformation being a transformation which acts upon a certain wave train (a light wave train, in the most general case), it has no consequences upon the age of two observers moving on closed-loop trajectories. So no contradiction can appear when the two observers are meeting again.

\subsection{Possibilities of using the principle of least action in connection with the wave-train interpretation}

We begin by writing the propagation equation for an electromagnetic wave inside an observer material medium under the form $d x^{2}+d y^{2}+d z^{2}=c^{2} d t_{2}$ ( $c$ representing the light speed). It results that $c^{2} d t^{2}-d x^{2}-d y^{2}-d z^{2}=0$ for all points inside the material medium where the wave has arrived. But

$$
c^{2} d t^{2}-d x^{2}-d y^{2}-d z^{2}=d s^{2},
$$

where $d s$ is the cuadridimensional space-time interval. The propagation equation of the optical wave can be written as $d s=0$, and so it results that the trajectory of the wave inside the material medium between two points $a$ and $b$ is determined by the equation

$$
\int_{a}^{b} d s=\Delta s=0
$$

By the other hand, for mechanical phenomena the quantity determining the trajectory of a material body inside a reference system is the action $S$. Under a relativistic form, it can be written as $S=-m c \int_{a}^{b} d s, m$ representing the mass of the body, and $a, b$ the space-time coordinates for two points situated along the "universe line" on which the body moves. The principle of least action can be written as $\delta S=-m c \delta \int_{a}^{b} d s=0$.

While $\delta S=\sum_{i} m c u_{i} \delta x_{i}$, where $u_{i}=v_{i} / \sqrt{1-v^{2} / c^{2}}$ for $i=1,2,3$ and $u_{4}=$ $i c / \sqrt{1-v^{2} / c^{2}}$, it results finally that $\sum_{i} p_{i}^{2}=-m^{2} c^{2}, p_{i}$ being the cuadrivector $\partial S / \partial x_{i}$ (the momentum). For a free particle, $p_{i}=m u_{i}$. It can be noticed that the infinite small cuadridimensional interval $d s$ is used both for describing the propagation of an electromagnetic wave and the movement of a body inside a reference system. While is it related to the action $S$, this result is easy to be understood (the principle of least action being a basic principle in nature). The next step consists in pointing the fact that the previous integral $\Delta s=0$ (determining the trajectory of the optical wave train inside the material medium) is based on the supposition that both points $a, b$ belong to the material medium (otherwise, the velocity of the wave may differ, depending on the dielectric and magnetic constants of the material). So the equation can be directly used in measurement procedures (for establishing trajectory or other properties of the wave only for the time interval when the optical wave train exists in that material medium [17]). If an observer has to analyze a wave train emitted in another material reference system, he must use the invariance property 
of the cuadriinterval: $d s=d s^{\prime}$, where $d s$ represents the cuadriinterval between two close events in a certain inertial reference system and $d s^{\prime}$ represents the cuadriinterval between the same two events measured in another reference system. While $d s=d s(d x, d y, d z, d t)$ is determined inside the observer reference system and $d s^{\prime}=d s^{\prime}\left(d x^{\prime}, d y^{\prime}, d z^{\prime}, d t^{\prime}\right)$ corresponds to the reference system where the wave has been emitted, it results that the cuadridimensional interval $d s$ moves into the cuadridimensional interval $d s^{\prime}$ by a function

$$
d s(d x, d y, d z, d t) \Longrightarrow L \Longrightarrow d s^{\prime}\left(d x^{\prime}, d y^{\prime}, d z^{\prime}, d t^{\prime}\right)
$$

where the arguments of $d s$ are transformed by the Lorentz relations

$$
d x^{\prime}=\frac{d x+v d t}{\sqrt{1-v^{2} / c^{2}}}, \quad d y^{\prime}=d y, \quad d z^{\prime}=d z, \quad d t^{\prime}=\frac{d t+v d x / c^{2}}{\sqrt{1-v^{2} / c^{2}}}
$$

for $v$ parallel to $O x$ (all the space and time intervals $d x, d y, d z, d t$ being considered inside the observer material medium after the emitted optical wave train arrives), and $d s=d s^{\prime}$. The above relation can be considered as presenting a transformation of the received wave train (with $x, y, z, t$ coordinates) into a "supposed" wave train corresponding to the case when the wave train would not have entered inside the observer material medium. For determining the real trajectory of the wave before interaction the observer must extend the trajectory of the received wave train (having coordinates $x^{\prime}, y^{\prime}, z^{\prime}, t^{\prime}$ in the past and outside the observer material medium, using the relation

$$
\int_{a}^{b} d s^{\prime}=\Delta s^{\prime}=0
$$

\subsection{Non-Markov aspects of pulse transformation}

We have also to emphasize the non-Markov aspect of Lorentz transformation which acts upon a received wave train when this interacts with the observer material medium. At the initial moment of time (the zero moment) we can consider that new values for wave quantities are generated as a result of the Lorentz matrix action upon the received values (cuadrivectors or cuadritensors). This represents a Markov transformation (using some physical quantities defined at a certain moment of time $t=0$, we can obtain the result of that transformation at a time moment $t+d t=0+d t$ ).

Yet if we analyze the wave train transformation at a subsequent moment of time (after the zero moment when the wave was received) we can notice that the physical quantities corresponding to cuadrivectors and cuadritensors are not just modified (by the action of Lorentz matrix) but are also translated at a different time moment (according to Lorentz formulae for transforming space-time coordinates). This implies that the physical quantities corresponding to the transformed wave train (defined in the observer material reference system) depend on the physical quantities corresponding to the unchanged wave train (supposed situation) at a previous time moment. Not being possible to use values of certain quantities at a time moment $t$ for obtaining the values of that physical quantities at a time moment $t+d t$ for $t>0$, it results that the Lorentz transformation of a received wave train (an electromagnetic or optic pulse or an associated wave corresponding to a particle) is a non-Markov transformation. In future studies, this aspects should be studied using aspects connected to time series inside a material medium [18]. 


\section{Conclusions}

This study has shown that certain intuitive problems connected with measurements of sequences of pulses on closed-loop trajectories in special relativity and noncommutative properties of operators in quantum physics imply a more rigorous definition of measurement method and of the interaction phenomena (classified according to a possible memory of previous measurements), so as to avoid logical contradictions due to a possible resynchronization. It is also shown that the use of the least action principle requires a specific space-time interval available for a space-time measurement in an implicit form. Due to this, it results in a certain distinction between the set of existing space-time intervals (which can be defined on unlimited space-time intervals) and the set of measured space-time intervals (established using measuring methods based on waves and always defined on limited spacetime intervals).

\section{References}

[1] E. Bakhoum, "Fundamental disagreement of wave mechanics with relativity," Physics Essays, vol. 15, no. 1, pp. 87-100, 2002.

[2] P. A. M. Dirac, The Principles of Quantum Mechanics, Oxford University Press, Oxford, UK, 1958.

[3] R. Feynman, The Feynman Lectures on Physics, vol. 2, Addison Wesley, Reading, Mass, USA, 1964.

[4] Y. Aharonov and D. Bohm, "Significance of electromagnetic potentials in the quantum theory," Physical Review, vol. 115, no. 3, pp. 485-491, 1959.

[5] Y. Imry and R. A. Webb, "Quantum interference and the Aharonov-Bohm effect," Scientific American, vol. 260, no. 4, pp. 56-62, 1989.

[6] A. P. French and E. F. Taylor, An Introduction to Quantum Physics, Norton Publications, New York, NY, USA, 1978.

[7] L. de Broglie, New Perspectives in Physics, Basic Books, New York, NY, USA, 1962.

[8] L. de Broglie, The Current Interpretation of Wave Mechanics: A Critical Study, Elsevier, Amsterdam, The Netherlands, 1964.

[9] D. Grifiths, Introduction to Elementary Particles, John Wiley \& Sons, New York, NY, USA, 1987.

[10] C. Morarescu, "Inner potential of generating pulses as a consequence of recurrent principles and specific computing architecture," in Computational Science and Its Applications, vol. 3980 of Lecture Notes in Computer Science, pp. 814-820, Springer, Berlin, Germany, 2006.

[11] C. Toma, "A connection between special relativity and quantum theory based on non-commutative properties and system-wave interaction," Balkan Physics Letters, vol. 5, pp. 2509-2513, 1997.

[12] M. Takeda, Sh. Inenaga, and H. Bannai, "Discovering most classificatory patterns for very expressive pattern classes," in Discovery Science, vol. 2843 of Lecture Notes in Computer Science, pp. 486-493, Springer, Berlin, Germany, 2003.

[13] C. Toma, "The advantages of presenting special relativity using modern concepts," Balkan Physics Letters, vol. 5, pp. 2334-2337, 1997.

[14] C. Cattani, "Harmonic wavelets towards the solution of nonlinear PDE," Computers \& Mathematics with Applications, vol. 50, no. 8-9, pp. 1191-1210, 2005.

[15] M. Simeonidis, S. Pusca, G. Toma, A. Toma, and T. Toma, "Definition of wave-corpuscle interaction suitable for simulating sequences of physical pulses," in Computational Science and Its Applications, vol. 3482 of Lecture Notes in Computer Science, pp. 569-575, Springer, Berlin, Germany, 2005.

[16] P. Sterian and C. Toma, "Methods for presenting key concepts in physics for MS students by PhotonMD program," Bulgarian Journal of Physics, vol. 27, no. 4, pp. 27-30, 2000.

[17] C. Toma, "The use of the cuadridimensional interval- the main possibility for improving the Lorentz formulae interpretation," in Proceedings of the European Conference on Iteration Theory (ECIT '97), vol. 2, p. 202, Pitesti, Romania, November 1997.

[18] M. Olteanu, V.-P. Paun, and M. Tanase, "The analysis of the time series associated to sem microfractographies of Zircaloy-4," Revista de Chimie, vol. 56, no. 7, pp. 781-784, 2005. 


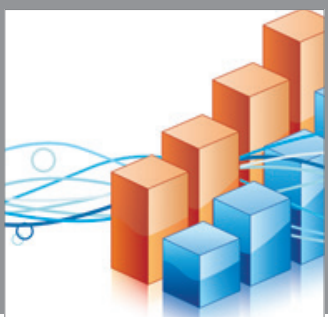

Advances in

Operations Research

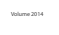

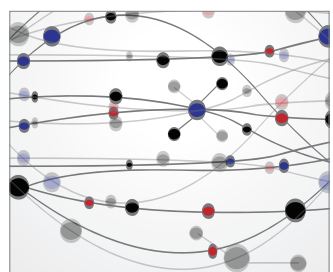

\section{The Scientific} World Journal
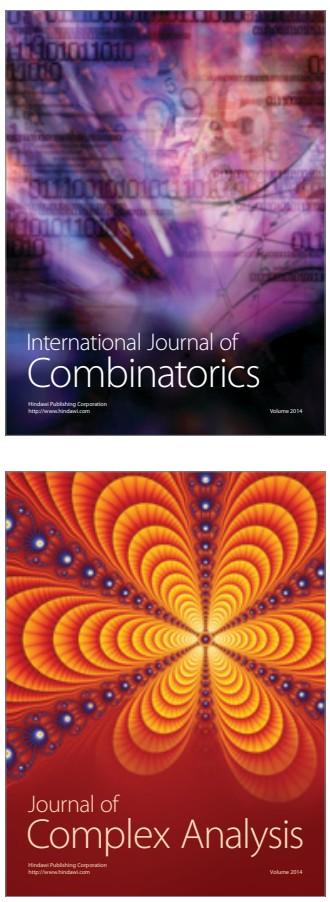

International Journal of

Mathematics and

Mathematical

Sciences
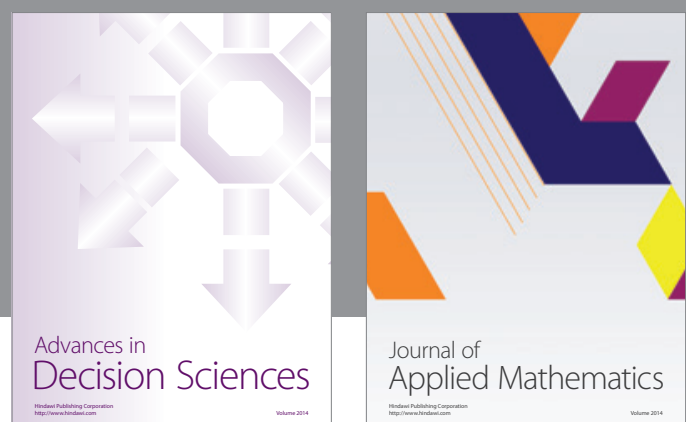

Journal of

Applied Mathematics
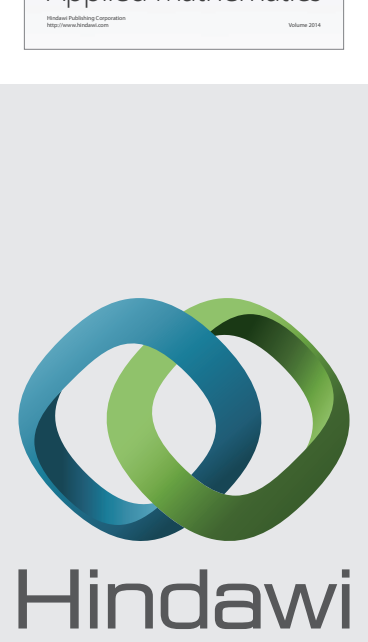

Submit your manuscripts at http://www.hindawi.com
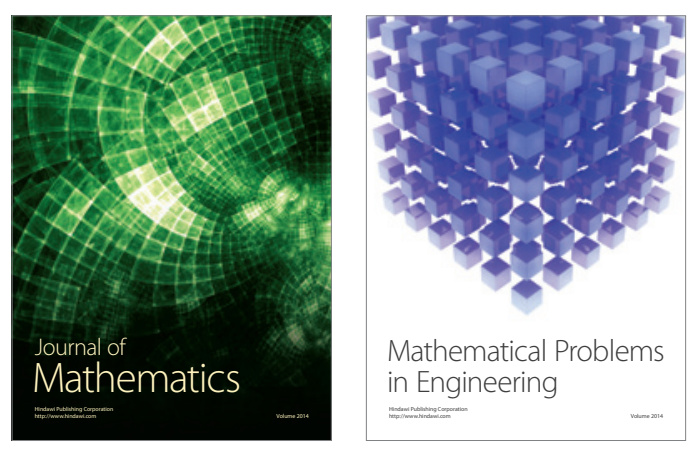

Mathematical Problems in Engineering
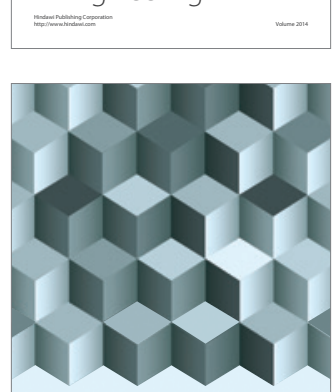

Journal of

Function Spaces
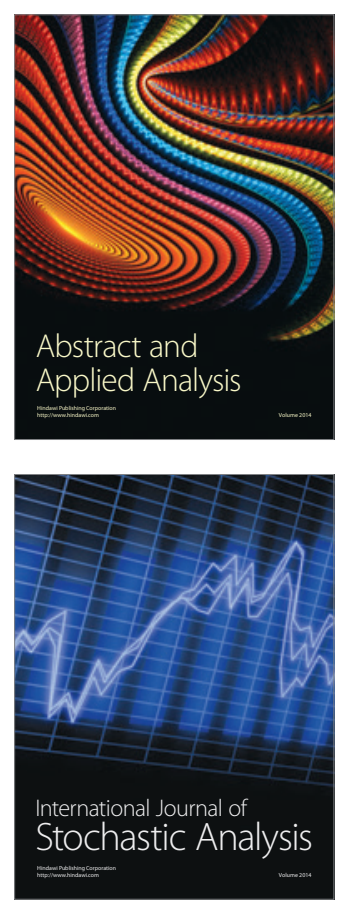

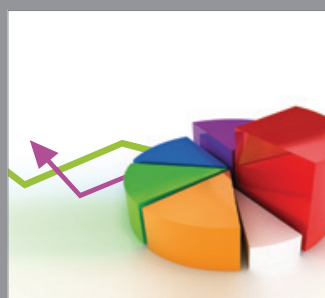

ournal of

Probability and Statistics

Promensencen
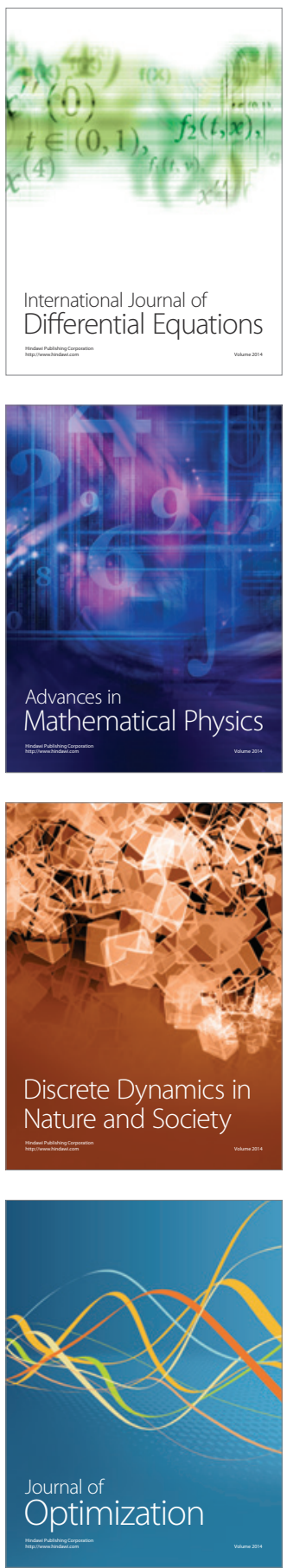